\title{
EBSD Analysis of Orientation Gradients Developed near Grain Boundaries
}

\author{
Natalia Soledad De Vincentis ${ }^{1 *}{ }^{\oplus}$, Analía Roatta ${ }^{1,2}$, Raúl Eduardo Bolmaro ${ }^{1,2}$, $^{-}$ \\ Javier Walter Signorelli, ${ }^{1,2}$ \\ ${ }^{1} I n s t i t u t o$ de Física Rosario - IFIR - UNR - CONICET, Ocampo y Esmeralda, Rosario, 2000, Santa Fe, \\ Argentina \\ ${ }^{2}$ Facultad de Ciencias Exactas, Ingeniería y Agrimensura - FCEIA-UNR, Avenida Pellegrini 250, \\ Rosario, 2000, Argentina
}

Received: June 13, 2018; Revised: October 17, 2018; Accepted: November 16, 2018

\begin{abstract}
The local misorientation and orientation gradient development near grain boundaries (GBs) are analyzed in a deep drawing quality steel sheet (AKDQ) subjected to interrupted tensile tests in a notched sample. The microstructure is studied using electron backscatter diffraction (EBSD) with subgrain-level spatial resolution. The evolution of misorientation accumulation for particular GBs was traced in grains located in different zones inside the notch, identifying the effective area of influence of GBs inside the neighboring grains. A local study was performed, and the evolution in misorientation development near GB was investigated. The results show a low correlation between GB width and sharpness of the orientation gradient with the mesoscopic strain, but instead orientation gradients between GB zones and the interior of the grains were observed with increasing strain. The increase in severity observed in some GBs can be related to dislocation pile up development, which reduces the permeability of a boundary to dislocation transmission.
\end{abstract}

Keywords: Orientation gradient, EBSD, Plastic deformation, Slip transmission.

\section{Introduction}

Plastic strain methods, such as stretching and deep drawing, are of great interest in materials processing. Such processes, however, lead to a heterogeneous local orientation development, which is a consequence of dislocation accumulation that can lead to rotations of the crystalline matrix. It has been investigated whether this misorientation development is somehow related to other microstructural characteristics such as grain size ${ }^{1,2}$ or shape ${ }^{3}$, or crystal orientation ${ }^{4,5}$. In polycrystalline $\mathrm{Cu}$ with grains in the order of $50 \mu \mathrm{m}$ that underwent plastic deformation, Soifer et al. ${ }^{6}$ found that hardness in zones up to $2 \mu \mathrm{m}$ from grain boundaries was $50 \%$ higher than at the inner area of the grains. A similar behavior was observed by Soer et al. ${ }^{7}$ in Mo bicrystals in $1 \mu \mathrm{m}$ wide zones near GB. According to the mentioned papers, there is evidence of a certain area inside the grains and near GBs with different characteristics from the rest of the grain, which can be considered as an "area of influence" of the GB inside a grain. Calcagnotto et al. ${ }^{8}$, on the other hand, reported orientation gradients near ferritemartensite interfaces in dual-phase steel. It is important to highlight that the presence of orientation gradients takes high relevance during recrystallization processes, grain fragmentation and stress concentration, particularly near the GBs, which can then lead to fractures ${ }^{9,10}$. Basu et al. ${ }^{11}$ determined the local average misorientation profile inside

*e-mail: devincentis@ifir-conicet.gov.ar a particular grain starting from one of its grain boundaries in Ti; they observed that the local misorientation has a local minimum near the grain boundary and then increases up to a maximum value and decreases as the data in the calculation are deeper in the core area of the grain. Mishra et al..$^{12}$ worked with aluminum samples deformed under tension and observed higher local misorientation near GBs, which then decreased with no particular behavior up to a point where it became nearly constant, while Allain-Bonasso et al. ${ }^{1}$ and Subedi et. al. ${ }^{13}$ detected that misorientation decreased linearly from the GB into the inner part of the grain in IF steel and copper subjected to uniaxial tension. Other authors have performed indentation tests on both sides of selected grain boundaries in order to characterize the obstruction to slip provided by the interface ${ }^{6,14,15}$. More recently, the misorientation accumulation near GB using EBSD for a drawing quality steel under four different in-plane loading conditions was characterized through two EBSD-based parameters: gradient average severity (GAS) and boundary effective thickness (BET). This model was found to be valid for approximately $50 \%$ of the GB segments, and showed that the GAS parameter evidenced good correlation with macroscopic strain, while BET values resulted in 1.5-2 $\mu \mathrm{m}$ for all cases, representing the width of influence of GBs inside a grain ${ }^{16}$.

The observed misorientation accumulation near GBs and its relationship with dislocation transmission through GBs are thoroughly discussed. According to Aust and Chen ${ }^{17}$, slip activation can be defined as "the process where slip- 
bands from one crystal can initiate or activate slip-bands in an adjacent crystal". Four possible interactions between a dislocation and a GB can occur, amongst which types (ii) and (iii) happen more often ${ }^{18,19}$ :

i. direct dislocation transmission through cross-slip;

ii. direct transmission but with residual dislocations contributing to the GB;

iii. indirect transmission, where incident and transmitted slip systems do not intersect, and with residual dislocations at GB - could be dissociated into two stages, where the incident dislocation is incorporated at the GB and then a different dislocation is emitted into the neighboring grain along another slip system;

iv. no transmission - the dislocation would pile-up at the GB or become part of it.

While assessing the mentioned conditions, several criteria for slip transfer through GB have been developed. One of the first models was proposed by Livingston and Chalmers ${ }^{20}$, and was based in geometric considerations between slip systems at both sides of the GB. Shen et al. ${ }^{21}$ added that the resolved shear stress of the transmitted slip system should be maximized, and Lee et al. ${ }^{14}$ considered that a third condition must be met: the residual dislocation at the GB should be minimized. Van Beers et al. ${ }^{22}$ proposed that plastic slip at GB is determined by a competition between three interface microforces: a bulk-induced microforce, related to bulk GND densities; a dissipative microforce, associated with the resistance to slip at the interface, and an energetic microforce related to the GB net Burgers vector, which tends to oppose accumulation of interface residual defect. Higher misorientation of neighboring grains delays interface normal slip and reduce slip rates, increasing strain-hardening rates. At smaller grain sizes, larger strain gradients and higher GND densities are developed, causing the initiation of interface slip to occur at lower shear strain. Abuzaid et al. ${ }^{23}$ also correlated the plastic strain across GBs with the residual Burgers vector, and Vachhani et al ${ }^{24}$ reported a $10 \mu \mathrm{m}$ wide hardening area near GB related to dislocation accumulation. Other authors analyzed how the alignment of slip systems activated at both sides of a GB determine the feasibility of slip transmission ${ }^{14,21,25-27}$. They consider that the permeability of a particular GB is related with the angles between slip vectors ( $\kappa)$, between slip plane normals $(\psi)$ and between the two slip plane intersections with the GB plane $(\theta)$. Two of the mentioned angles can be obtained easily from 2D orientation analysis, while the determination of $\theta$ requires the knowledge of the GB plane, which can only be assessed by destructive methods (3D EBSD - FIB for example ${ }^{28,29}$ ). Nonetheless, authors like Bieler et al. ${ }^{27}$ attempted to work from the knowledge of $\kappa$ and $\psi$, maximizing the following permeability factor:

$$
\chi=\cos (k) \cos (\psi)
$$

The authors correlated this value with Schmid factors for particular GBs, obtaining good correlation in some cases, implying that the third angle would provide very valuable information to assess slip transfer conditions.

In this work the EBSD technique is used to determine the evolution of orientation gradients near grain boundaries in a steel sheet deformed by interrupting the tension tests. This procedure allowed to follow the misorientation developed in particular GBs, which brought information on the evolution of the parameters related to crystallographic orientation with increasing strain. The estimation of the severity of the orientation gradient and area of influence of GBs and their permeability at different deformation stages lead to the identification of possible interactions between dislocations and GBs.

\section{Experimental Methods}

\subsection{Material and characterization techniques}

Samples were taken from a $0.67 \mathrm{~mm}$ thick AluminumKilled Drawing Quality (AKDQ) steel sheet. Composition is presented in Table 1. Average grain size of $5-10 \mu \mathrm{m}$ and a Vickers hardness of 106HV5 were measured at as-received condition. A sample was laser-cut from this sheet in order to perform uniaxial tension tests, as represented in Fig. 1. A notch was performed near the half-length of the sample, which allowed concentrating the strain developed in this area, and also caused different strain levels across the notch. Small pyramidal indents were used to delimit different zones inside the notch area, allowing the easy localization of each particular zone after deformation.

The tension tests were performed in an Instron 5989 with $150 \mathrm{kN}$ capacity and at a head displacement of 8.3 $10^{-4} \mathrm{~mm} / \mathrm{s}$. In order to evaluate the microstructural changes during deformation, the sample was subjected to a tensile test performed in two stages: one of them was interrupted after 100 s and the other one after 300s (loads and displacements shown in Fig. 2). EBSD scans were taken from the selected areas inside the notch in as-received condition and after each test interruption. It must be taken into account that the displacement values shown in Fig. 2 correspond to the macroscopic (average) strains for the tensile sample, which differ from the mesoscopic strains experienced inside each area in the notch, as will be shown ahead.

EBSD patterns measurements were performed in an FEI Quanta E scanning electron microscope with field emission gun and EBSD detector with phosphor screen and DigiView camera. Surface preparation of the sample was performed using grinding paper in order to obtain a plain surface, then $9,6,3$ and $1 \mu \mathrm{m}$ diamond paste and $0.05 \mu \mathrm{m}$ colloidal silica. The sample was polished only in the as-received condition, in an attempt to preserve the indentation marks that were performed in it to delimitate the different zones. 
Table 1. Chemical composition of the AKDQ steel sheet used (excluding surface covering) $( \pm 0.001)$.

\begin{tabular}{cccccccccccc}
\hline Element & $\mathrm{C}$ & $\mathrm{P}$ & $\mathrm{S}$ & $\mathrm{Mn}$ & $\mathrm{Si}$ & $\mathrm{Cr}$ & $\mathrm{Ni}$ & $\mathrm{Cu}$ & $\mathrm{Mo}$ & $\mathrm{Al}$ & $\mathrm{N}(\mathrm{ppm})$ \\
\hline \% weight & 0.070 & 0.020 & 0.009 & 0.300 & 0.040 & 0.020 & 0.010 & 0.010 & 0.010 & 0.192 & 88 \\
\hline
\end{tabular}

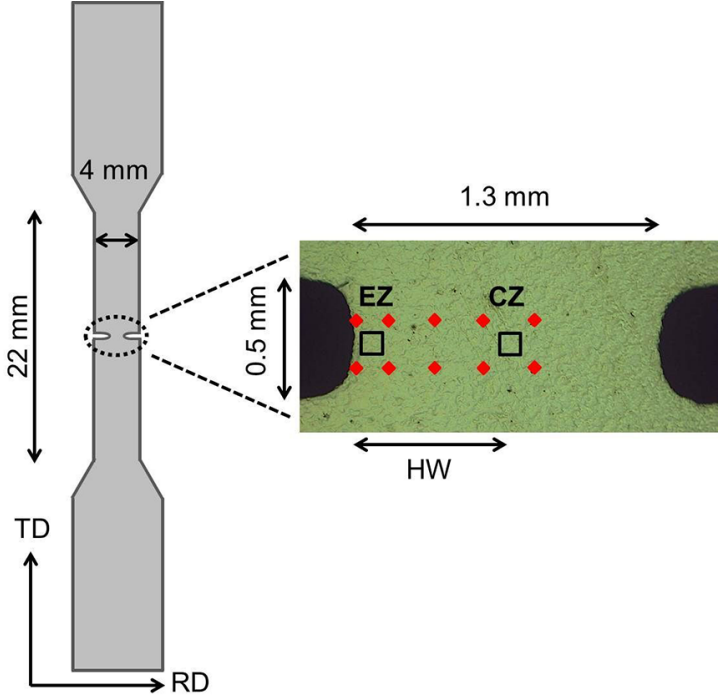

Figure 1. Sketch of the notched tensile sample indicating the different zones. HW is half the width of the notch.

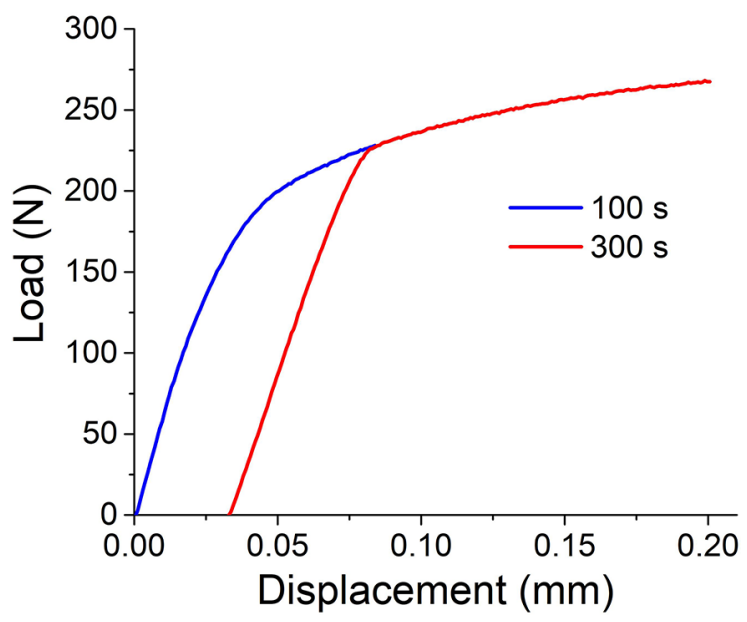

Figure 2. Load vs. Displacement curves obtained from both deformation stages.

EBSD scans were measured using OIM 5 software with $10 \mathrm{keV}, 0.1 \mu \mathrm{m}$ step size (hexagonal grid) over areas of 80 $\mu \mathrm{m} \times 80 \mu \mathrm{m}$ approximately, and the studied area enlarged with increasing deformation in order to comprise the same grains. No clean-up routine was used.

\subsection{Definition of region of influence of grain boundary}

The model used in this research concurs with the observations made by several authors ${ }^{1,13}$, where a decrease in misorientation was observed from the GB into the grain interior, in some cases following a straight line with negative slope ${ }^{1}$. This distinctive behavior of crystal misorientation near GB would allow identifying two separate zones inside the grain: the GB zone (GBZ), area close to the GB where a generally decreasing orientation gradient is observed, and the inner or core zone, where at first no particular misorientation profile is expected. This is schematized in Fig. 3. It must be stated that each GBZ can be different at both its sides, and might also differ between all the boundaries of a certain grain.

A predefined GBZ analysis is considered for each GB, consisting in an area situated at each side of the analyzed GBs which included all the pixels at a distance less than a given threshold from the GB, and no closer to any other GB. The procedure consisted in the following steps (see ${ }^{16}$ for details):

1. The pixels at the GB are identified; these pixels have first neighboring pixels that do not belong to the same grain than them;

2. The GB pixels are separated into subsets $\mathrm{GB}_{\mathrm{i}}$, which correspond to each border with a neighboring grain;

3. The GBZs are determined by evaluating the distance between each pixel in the grain and each GB pixel; in the present case the threshold is set to $5 \mu \mathrm{m}$. These pixels are considered to belong to the GB neighboring zone, being this boundary for which the smallest distance is obtained.

These GBZs are the areas near GBs that were inspected for possible orientation gradient, the presence of which would define an admissible GBZ. Local average misorientation measurement was performed over $0.2 \mu \mathrm{m}$ thick bins starting at a GB and moving to larger distances using the method proposed by Kamaya ${ }^{30}$ :

$$
M_{L}(i)=\frac{1}{6} \sum_{j=1}^{6} m(i, j)
$$

where $m(i, j)$ represents the misorientation between pixels i and $j$, and the limit of $j$ can be less than 6 in case of pixels located near GBs.

The decreasing linear profile can be characterized using 2 parameters: $\lambda$ and $\beta$ (Fig. 4). The $\lambda$ parameter can be considered as the extension to which the GB has an influence inside the grain, while the value of $\beta$ factor can be associated with the severity of the GB misorientation, since $\operatorname{tg}(\beta)$ is the slope of the decreasing line. A value of $\operatorname{tg}(\beta)=1$ would imply a decrease in misorientation in $1 \% \mu \mathrm{m}$. Two other parameters of interest can be extracted from the misorientation profile: $Y_{0}$, which represents the intercept of the decreasing line with the $\mathrm{GB}$, and $\mathrm{M}_{\mathrm{C}}$, being an estimation of the misorientation in the area not belonging to the admissible GBZ ${ }^{16}$. Fig. 4 shows an example of the misorientation profile obtained from a GBZ after the $300 \mathrm{~s}$ tension test. A decrease in local 


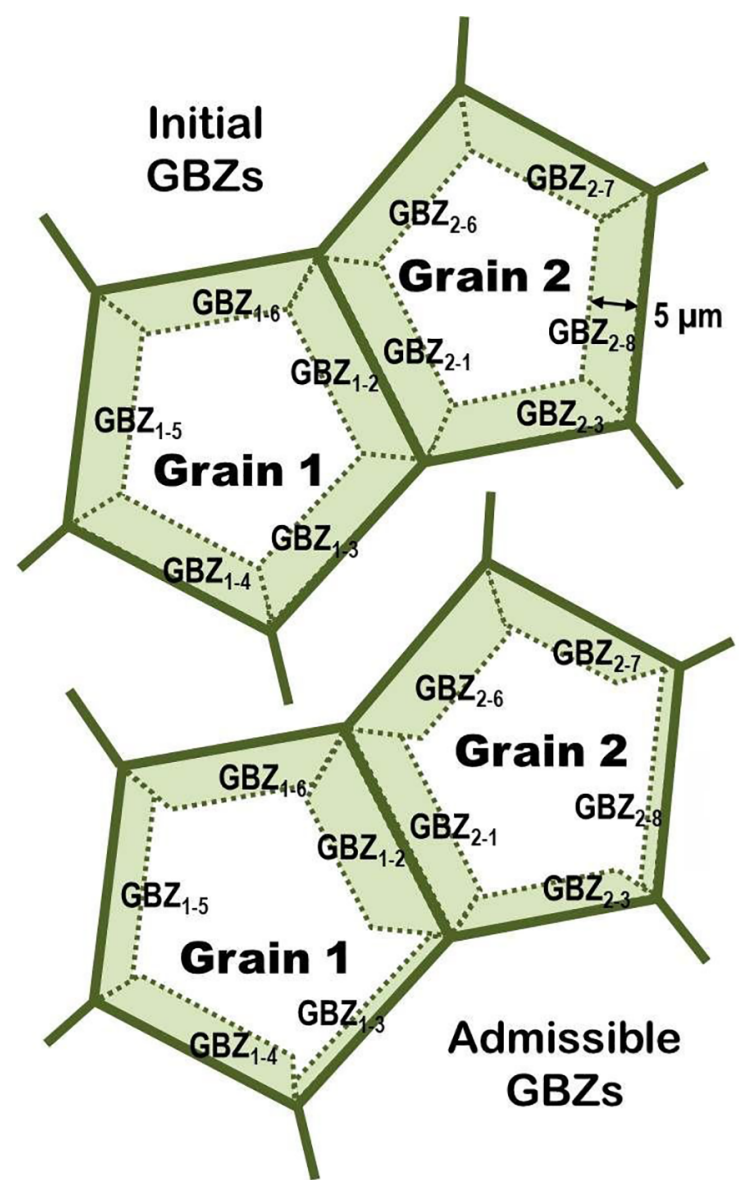

Figure 3. Schematic representation of the initial GBZ considered in the calculations and the admissible GBZs resulting from orientation gradient analysis.

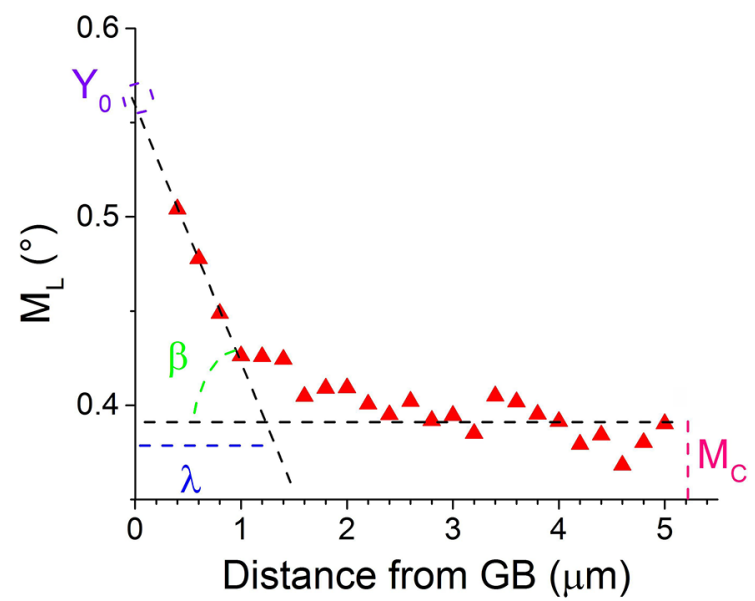

Figure 4. Misorientation profile through a particular GB in EZ after the $300 \mathrm{~s}$ test showing $\beta, \lambda, \mathrm{Y}_{0}$ and $\mathrm{M}_{\mathrm{C}}$ parameters.

misorientation can be observed from the GB to the inside of the grain, being almost linear, up until a point where it becomes almost constant; the admissible GBZ of this particular GB can be easily identified, around $1.4 \mu \mathrm{m}$ from the GB. The four mentioned parameters can be extracted from a least-squares-fitting of the experimental data obtained from 2D measurements. A more thorough analysis would require the $3 \mathrm{D}$ measurement of misorientation, which is beyond the capabilities of the methods used in this paper, as was stated earlier.

\section{Results}

\subsection{Plastic strain determination}

The characteristics of the experimental procedure were designed specifically in order to assess the evolution of orientation gradient on selected grain boundary zones with the magnitude of plastic strain induced in the material. The distribution of average plastic strain along the symmetry line of the notched area was measured by considering the relative motion of a set of Vickers microindentation marks (Fig. 1). These values were also complemented with calculations of relative motion of triple points, as proposed by Wagner et al. ${ }^{31}$, obtaining similar results. The strain values determined after each test interruption are presented in Fig. 5.

The effect of the notch is to concentrate plastic deformation at the notch tip comparing with the center of the sample, and also to change the type of solicitation; the triaxiality levels diminish when the areas close to the notch root are reached ${ }^{32,33}$.

After $100 \mathrm{~s}$, all four zones developed a von Mises equivalent strain in the range of approximately 3-6\% approximately, being larger for the zone farther from the sample center. Following the 300 s test, strain increased for all zones ranging from about $6 \%$ near the center of the sample to $16 \%$ in the farthest zone, showing a non-homogeneous strain variation that can be related to the different straining processes taking

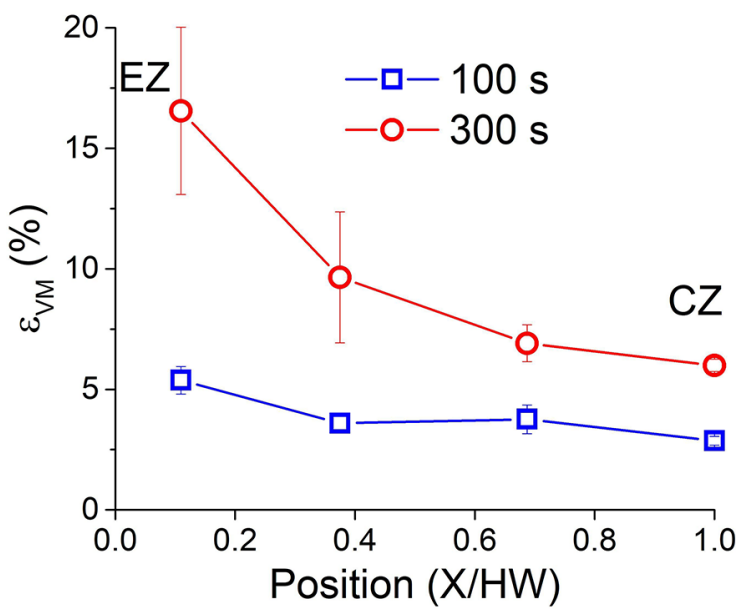

Figure 5. von Mises equivalent strain calculated for each zone - $\mathrm{X}$ represents the position relative to the edge of the notch. 
place. Elastoplastic Finite Element simulations of tension tests in notched specimens confirm the tendency of the results ${ }^{34}$.

\subsection{Misorientation profile near $G B$ and $G B$ permeability}

Inverse Pole Figure (IPF) maps obtained by EBSD for $\mathrm{CZ}$ (center zone) and EZ (edge zone) (see Fig. 1) after each test interruption are presented in Fig. 6 (initial misorientation, GB length and grain size in these zones as well as information regarding the intermediate zones can be found in the Supplementary Material). It can be seen that the scanned areas included few GBs, with highly valuable information of local misorientation development and its variation with increasing strain. The quality of the maps decreased at higher strain, being lower for EZ, which is the area closest to the edge of the notch. It must be taken into account that samples were only polished before the tensile tests and were not repolished afterwards, which implies that the increase observed in non-indexed pixels might be caused by the high misorientation developed under strain.

From local orientation data obtained by EBSD, local misorientation was calculated and plotted in Fig. 7. Up to $100 \mathrm{~s}$, both zones show similar proportion of misorientation boundaries (except for the streak observed in $\mathrm{CZ}$, region that is not involved in the calculation of the chosen grain boundaries), which tend to be located near GBs, although some other misorientation areas seem to appear inside the grains in EZ. After $300 \mathrm{~s}$, the amount of areas with misorientation gradient development increases considerably in EZ both near GBs and inside grains; strain localization starts to become evident and sub-grain boundaries appear. In both zones, grains were selected with the preference of having relatively long and straight GBs $(>10 \mu \mathrm{m})$.

The misorientation profiles obtained for the selected grain boundaries in $\mathrm{CZ}$ are presented in Fig. 8. Since the data obtained from the very first vicinity of the GB might carry an uncertainty caused by the overlapping of Kikuchi patterns from both neighboring grains, the points corresponding to $0.5 \mu \mathrm{m}$ at both sides from each GB are not considered for the calculations. At a first glance, it is obvious that although the selected boundaries correspond to the same grain $\mathrm{C}_{\mathrm{C}}$, the misorientation profile and its variation with strain are different for each neighbor. It can be observed also that increasing deformation lead to higher misorientation values, being the increase more appreciable when performing the $300 \mathrm{~s}$ test. In the section farther from the $\mathrm{GB}$ in grain $\mathrm{C}_{\mathrm{C}}, \mathrm{M}_{\mathrm{C}}$ values are different for each neighboring grain, indicating that either the misorientation of the inner region of the grain is not constant (although the differences might be covered by the uncertainties) or that the GBZ extends further than $5 \mu \mathrm{m}$.

Grain boundary $\mathrm{A}_{\mathrm{C}}-\mathrm{C}_{\mathrm{C}}$ (Fig. 8 (a)), for example, shows lower misorientation values on the side of grain $\mathrm{A}$ at the beginning and after $100 \mathrm{~s}$, while after $300 \mathrm{~s}$ the values increase up to similar values on both sides of the GB with very low
$0 \mathrm{~s}$
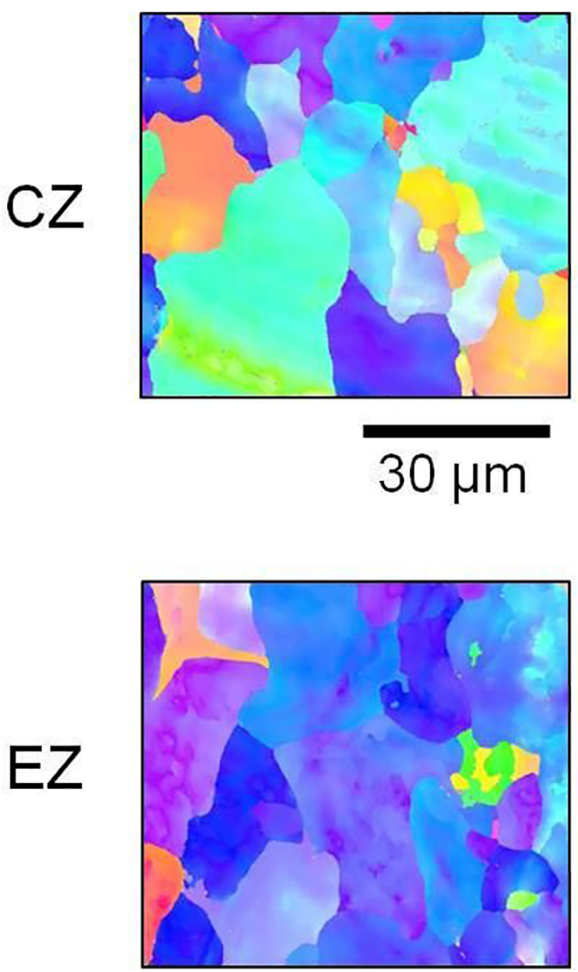

$100 \mathrm{~s}$
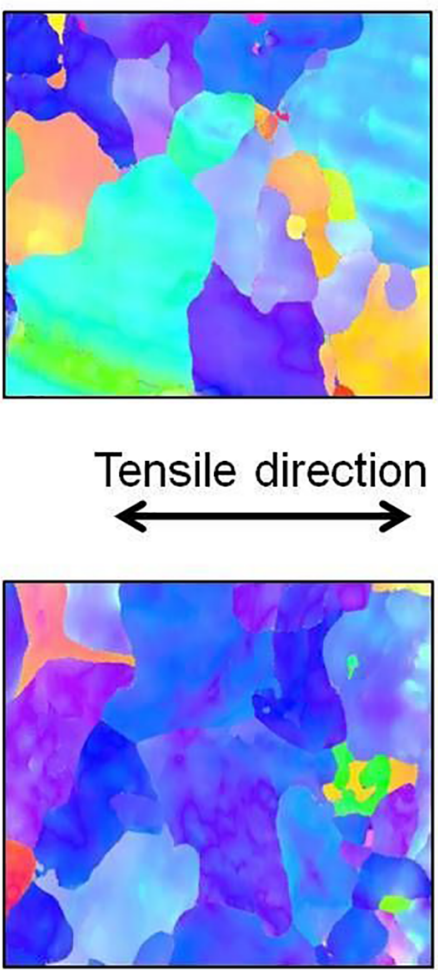

$300 \mathrm{~s}$
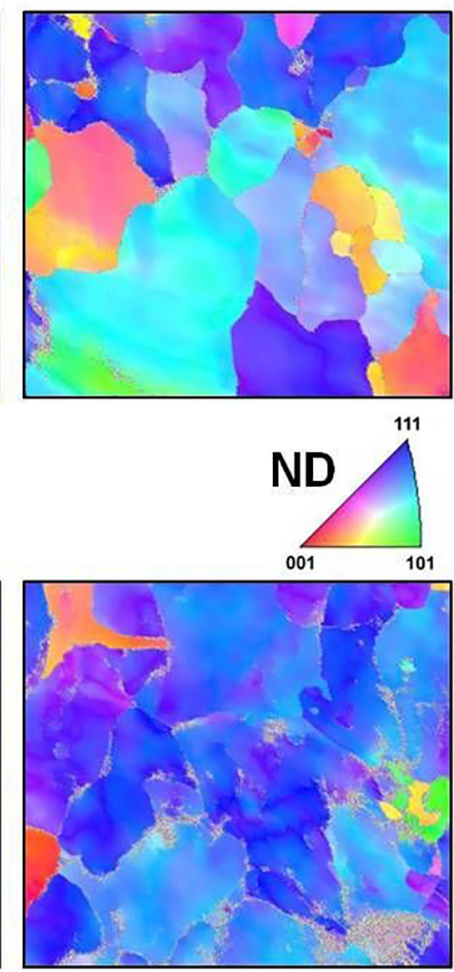

Figure 6. Inverse Pole Figure maps obtained from zones $\mathrm{CZ}$ and EZ after each test interruption. 


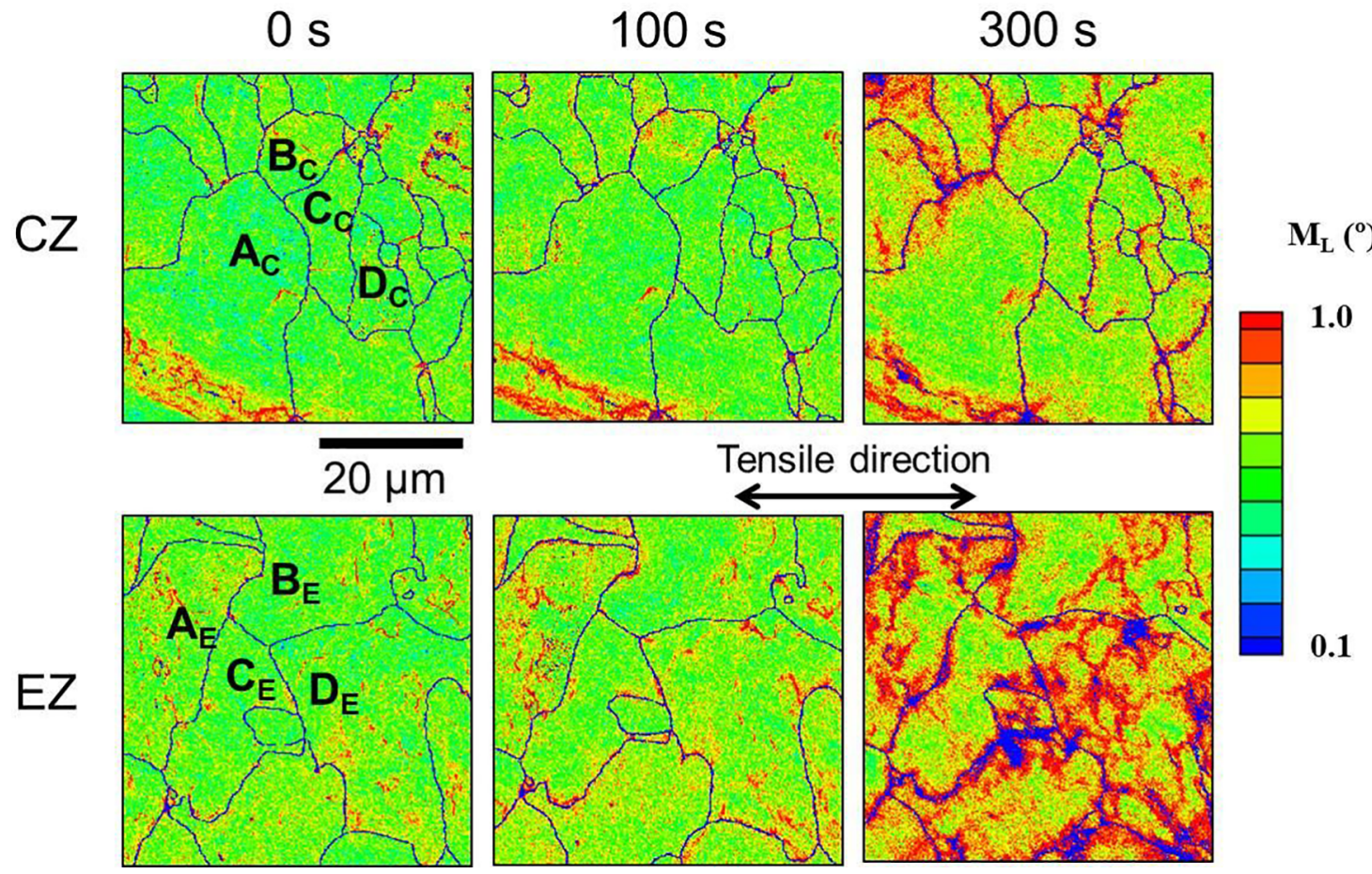

Figure 7. $\mathrm{M}_{\mathrm{L}}$ maps (logarithmic scale) obtained in $\mathrm{CZ}$ and $\mathrm{EZ}$ after each stage, indicating the grain boundaries to be analyzed.

gradient. At boundary $\mathrm{B}_{\mathrm{C}}-\mathrm{C}_{\mathrm{C}}$, lower misorientation can be appreciated at $\mathrm{C}$ side, with almost no increase after $300 \mathrm{~s}$, while an apparently uniform and higher misorientation is developed at B side. As in the previous GB, no appreciable gradient is observed; however, it is interesting to note that the points closest to the GB show less dispersion than those farther from it. $\mathrm{D}_{\mathrm{C}}-\mathrm{C}_{\mathrm{C}}$ boundary, on the other hand, evidences some degree of misorientation localization after $300 \mathrm{~s}$, being observed at both sides of the GB with different severity. On the side of grain $\mathrm{D}_{\mathrm{C}}$, localization of misorientation at the beginning and after $100 \mathrm{~s}$ is observed not immediately next to the GB but at $2 \mu \mathrm{m}$, and after $300 \mathrm{~s}$ misorientation increases all throughout the inspected $5 \mu \mathrm{m}$ length and presenting a linear decrease from the GB to the core of the grain, with an approximate severity of $1.43^{\circ}$. On $\mathrm{C}_{\mathrm{C}}$ side, misorientation at $100 \mathrm{~s}$ shows some localization at $1 \mu \mathrm{m}$, and after $300 \mathrm{~s}$ a larger misorientation gradient is evident near GB, being sharper than on $D_{C}$ under the same conditions $\left(\beta=2.86^{\circ}\right)$. It is interesting to note that in this particular GB an orientation gradient is obtained with increasing deformation, where no gradient was observed at the start.

The misorientation profiles obtained for selected GBs in EZ are presented in Fig. 9. It is evident that after $300 \mathrm{~s}$ the misorientation is larger for these GBs than for those corresponding to $\mathrm{CZ}$ after the same test, and even with larger severity and width of influence in some cases, which is compatible with a greater plastic deformation. The $100 \mathrm{~s}$ test, however, resulted in misorientations comparable to those obtained for $\mathrm{CZ}$ after $300 \mathrm{~s}$, both cases with similar von Mises strain.

All the selected GBs present different misorientation development. GB $A_{E}-C_{E}$ (Fig. 9 (a)) shows a different misorientation accumulation at both sides of the GB: while no gradient is observed in $\mathrm{A}_{\mathrm{E}}$ side at any strain degree, a slight misorientation accumulation is obtained en $\mathrm{C}_{\mathrm{E}}$ side after $100 \mathrm{~s}$ that then increases sharply after $300 \mathrm{~s}$ with a severity of $6.31^{\circ}$, where the maximum misorientation between both sides of the GB is approximately $0.2^{\circ}$. Not only the different misorientation profile at each side of the GB are of interest in this case, but also the fact that in grain $\mathrm{C}_{\mathrm{E}}$ an orientation gradient is obtained where there was no appreciable gradient at the start. $\mathrm{B}_{\mathrm{E}}-\mathrm{C}_{\mathrm{E}}$ boundary, on the other hand, shows similar misorientation up to $100 \mathrm{~s}$, but a different profile at both sides after $300 \mathrm{~s}$ : a low severity in grain $\mathrm{B}_{\mathrm{E}}\left(1.69^{\circ}\right)$ with a width of influence near $5 \mu \mathrm{m}$, and a more concentrated area of high misorientation in $\mathrm{C}_{\mathrm{E}}$ side, which does not behave as a straight decreasing line but that reaches a local minimum at approximately $2 \mu \mathrm{m}$. This GB might not be well fitted with the model proposed in this paper, but seems to correspond to the observations made by Mishra et al. ${ }^{12}$. GB $\mathrm{D}_{\mathrm{E}}-\mathrm{C}_{\mathrm{E}}$ seems to behave in a similar way to $A_{E}-C_{E}$ where an orientation gradient is observed after $300 \mathrm{~s}$ in $\mathrm{C}_{\mathrm{E}}$ grain $\left(\beta=5.99^{\circ}\right)$ but a nearly constant misorientation is observed in $\mathrm{D}_{\mathrm{E}}$, but in this case the misorientation values developed in this grain are higher and with more dispersion than in $\mathrm{C}_{\mathrm{E}}$.

Permeability values obtained for $\mathrm{CZ}$ and $\mathrm{EZ}$ are presented in Fig. 10. No appreciable change is registered with increasing 


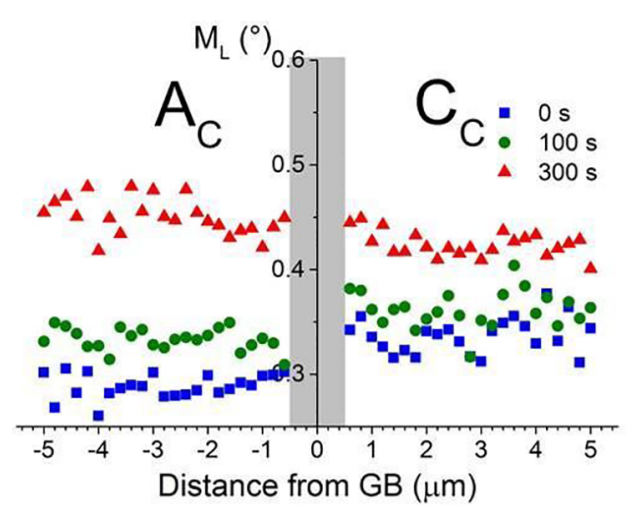

a

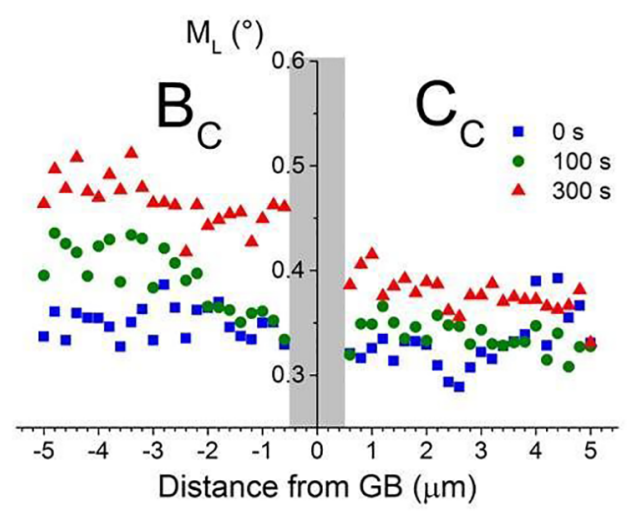

b

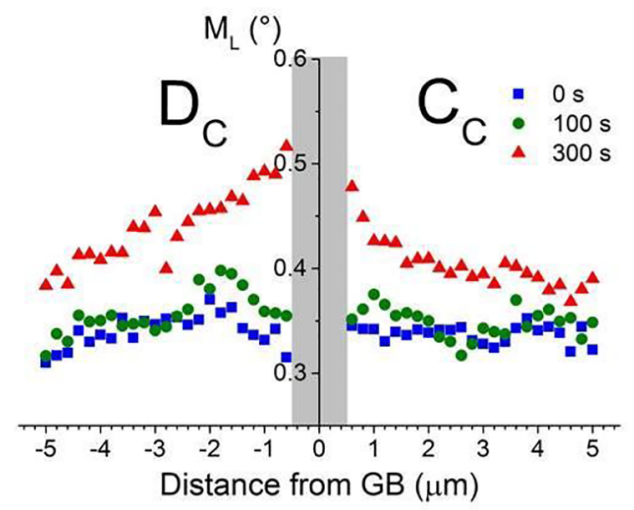

Figure 8. Misorientation profiles obtained for the chosen GBs in CZ.

strain in $\mathrm{CZ}$, which at first can be related to low strain values. $\mathrm{A}_{\mathrm{C}}-\mathrm{C}_{\mathrm{C}}$ seems to be the most permeable to slip transmission; GBs $B_{C}-C_{C}$ and $D_{C}-C_{C}$ show similar permeability but with very different misorientation localization near GBs, as was described above. In EZ, permeability values do not change appreciably up to $100 \mathrm{~s}$ for any $\mathrm{GB}$, being larger for $\mathrm{A}_{\mathrm{E}}-\mathrm{C}_{\mathrm{E}}$ and $\mathrm{B}_{\mathrm{E}}-\mathrm{C}_{\mathrm{E}}$, but an abrupt decrease is observed for the $300 \mathrm{~s}$ test in $A_{E}-C_{E}$. As was expressed above for this particular $\mathrm{GB}$, an orientation gradient was developed only on $\mathrm{C}_{\mathrm{E}}$ side at this strain level, which might imply that slip transmission through this GB was affected and dislocations have piled up from $C_{E}$ side, reducing the permeability of this boundary to dislocation transmission. In $\mathrm{B}_{\mathrm{E}}-\mathrm{C}_{\mathrm{E}} \mathrm{GB}$, however, although

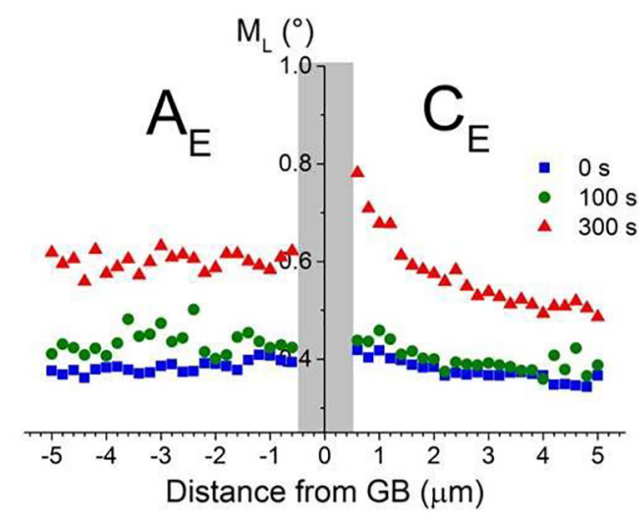

a

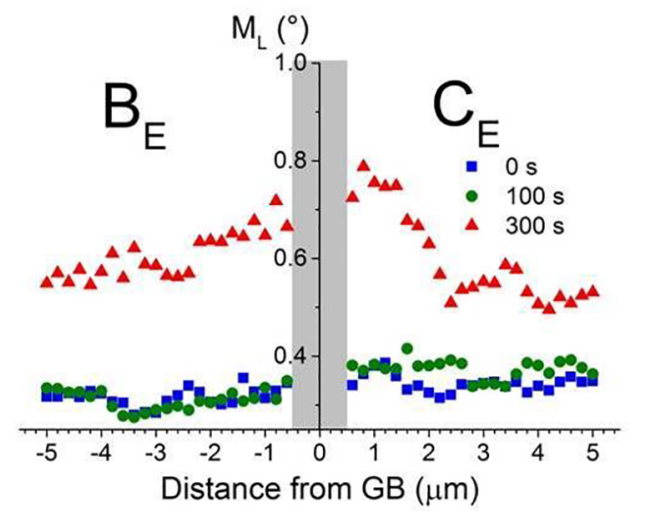

b

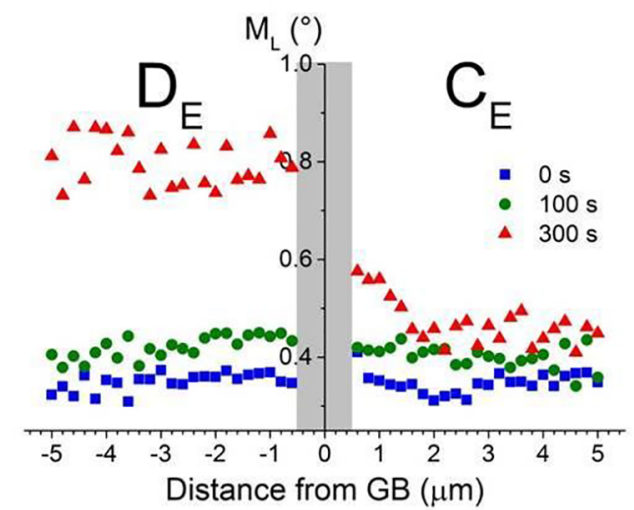

Figure 9. Misorientation profiles obtained for particular GBs in EZ.

different gradients are developed at each side of the GB in $300 \mathrm{~s}$, the permeability remains high, which might be related to the fact that misorientation at both sides of the $\mathrm{GB}$ is similar. The value obtained for $\mathrm{D}_{\mathrm{E}}-\mathrm{C}_{\mathrm{E}}$, on the other hand, decreases after $300 \mathrm{~s}$ but the initial value was already low (0.75); in this boundary not only gradient development but also misorientation values are different at both sides $\left(\Delta \mathrm{Y}_{0} \approx 0.2^{\circ}\right)$. It seems from these results that permeability for dislocation transmission through a GB is in some cases more sensitive to misorientation at both sides of a GB and in others to gradient development at both neighboring grains. The misorientation profiles and permeability factors of extra GBs in EZ can be found in the Supplementary Material. 


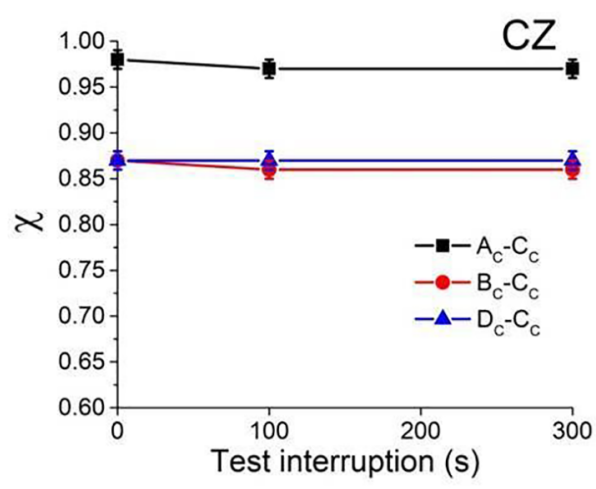

a

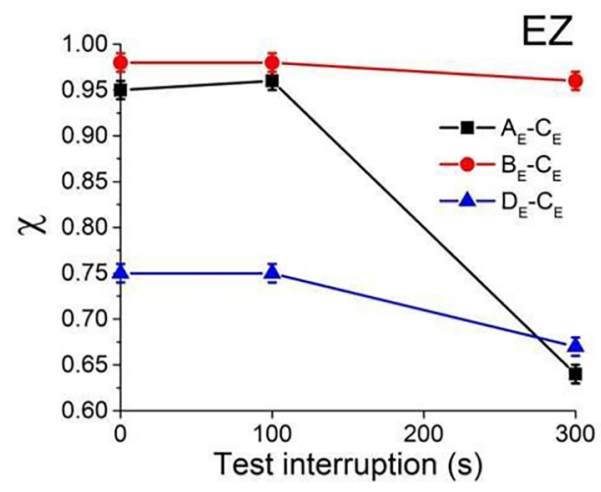

Figure 10. Highest permeability factors $\chi$ obtained for both zones.

Several remarks can be made regarding GB area of influence. The first comment must be devoted to the characteristics of the misorientation profile near GB. In this paper, the model that was used to characterize these profiles was proposed by Calcagnotto et al. ${ }^{8}$, in which the data close to the GB was fitted by a straight line with negative slope. This model assumes that misorientation is highest at the GB and decreases monotonically to the inside of the grain up to a certain misorientation, which in turn corresponds to a core misorientation which may or may not be somewhat homogeneous. In Figs. 7 and 8, however, it can be observed that in some cases the entire misorientation profile can be fitted by an exponential decay function; in spite of this, the model can still be used as an approximation, and has already been applied successfully in a previous paper where the same material was analyzed but the samples were only studied after being submitted to different straining conditions: uniaxial tension (UAT), plane strain (PS) and equibiaxial strain (EBA) ${ }^{16}$. In that case, the amount of GBs studied was significantly larger, and the statistical analysis performed allowed the determination of the two parameters mentioned earlier (GAS and BET), which are related to the the distributions of $\mathrm{Y}_{0}$ and $\lambda$ values. The strains involved in the corresponding experiments were about 0.4 and 0.8 von Mises, which are significantly larger than those involved in the present study, but even in that case the amount of GBs that could be characterized by this model was about $58 \%$ for UAT and PS and $47 \%$ for EBA. The results obtained in that paper state that the average severity (GAS) increased with larger macroscopic strain, while the effective thickness (BET) did not show appreciable variation with strain, and was near 1.5 - $2 \mu \mathrm{m}$. In the present paper, however, no particular correlation can be observed between the severity and width of GBZs and microscopic strain.

The method employed for the calculation of misorientation profiles involved the averaging of all the misorientations in the pixels located at the band $(\mathrm{i}, \mathrm{i}+0.2 \mu \mathrm{m})$ from the GB, but in cases where misorientation variations from point to point parallel to the GB are large, the average would be affected. Subedi et al. ${ }^{13}$ observed that in $\mathrm{Cu}$ samples deformed by tension, misorientation changed more drastically along the direction perpendicular to the GB than parallel to the GB; this result might imply that the heterogeneity in misorientation parallel to the GB could be negligible. This situation might also be one of the reasons why the dispersion observed in misorientation values was lower near GBs than farther from them.

c paper is GB permeability to slip transmission, which was carried out through the calculation of the $\chi$ parameter ${ }^{27}$. Analyzing each GB in particular, it can be observed that there is no significant variation in $\chi$ values with increasing deformation in CZ, but only in some cases in EZ. These results lead to conclude that strain and permeability for dislocation transmission through grain boundaries can only be related under high strain conditions. It must be noted that the mentioned strain is characteristic of the particular zone, whilst it is the local strain accumulation that should bear a relationship with GB permeability. In order to incorporate another validation of the results obtained, Schmid and Taylor factors were determined for the grains involved in the calculations; however, no relation could be found between them and $\chi$ factor nor with $\beta$ and $\lambda$ factors. Besides, according to the calculations performed by Bieler in the mentioned paper, the slip systems with the highest $\chi$ value not necessarily correspond to the largest Schmid factor.

As was mentioned in the Introduction, there are 4 possible interactions between an incident dislocation and a GB. Amongst the studied GBs, some of these particular cases can be identified. $\mathrm{GB} \mathrm{A}_{\mathrm{C}}-\mathrm{C}_{\mathrm{C}}$, in $\mathrm{CZ}$, seems to be a clear example of case I - direct dislocation transmission, because misorientation at both GBZs is similar and no local gradient is observed. In addition, the permeability factor is relatively high, which implies that slip systems at both sides are well aligned, allowing direct dislocation transmission. On the other hand, $G B A_{E}-C_{E}$, in EZ shows orientation gradient only on one side of the GB after 300 s, along with a sharp decrease in permeability, characteristics of case IV - no dislocation transmission. These results indicate that both approaches used in this paper - orientation gradient and permeability analyses - provide complementary information regarding the general 
behavior of dislocations approaching a GB. It must be taken into account that the $\chi$ factor calculated in the present paper involves only the angles between slip planes and directions at both sides of the GB, being relatively "incomplete". There is a factor similar to $\chi$ that takes into account not only the angles between slip directions at both sides of the GB but also the angle between the intersections of slip planes at both sides with the GB plane. This calculation is not performed in this paper because the GB plane inclination cannot be determined through $2 \mathrm{D}$ analysis. It would be interesting to pursue a pseudo-3D analysis in order to determine the plane inclination, which requires the sample to be cut and polished at a surface perpendicular to the analyzed surface and intersecting the grain boundary of interest; this analysis, however, would destroy the boundary and would not allow any following deformation process, therefore cancelling the possibility to follow-up local deformation at any particular boundary. In any case, the version of the $\chi$ factor applied in this paper sufficed to characterize the GB permeability to dislocation transmission and to identify some particular cases, when being combined with misorientation gradient analysis near GB.

\section{Conclusions}

Misorientation development near GB was analyzed using EBSD in a sample of AKDQ steel deformed by uniaxial tension test. The misorientation evolution was traced along particular GBs in zones near the center of the notch and near its edge, characterized by different mesoscopic strains.

A misorientation gradient profile developed near some GBs, defining an effective area of influence of the GB that can be characterized by the severity and width of the gradient. No simple correlations between the mentioned parameters and mesoscopic strain could be established, although general misorientation increased with larger strain. GB permeability to slip transmission changed with increasing strain only in particular boundaries in the area near the edge of the notch, suggesting the existence of a threshold strain that could result in appreciable variation in the permeability factor. In these cases, slip transmission through GBs was affected and dislocations piled up near the GB in the grains with larger severity, hence reducing the permeability of this boundary to dislocation transmission.

\section{Acknowledgments}

Authors would like to thank V. Tartalini, P. Risso and Ph.D M. Avalos for their help in sample preparation and camera optimization for EBSD.
The research leading to these results was funded by projects ANPCyT PICT 2013-0558 and Ecos Sud - MINCyT A16E03.

\section{References}

1. Allain-Bonasso N, Wagner F, Berbenni S, Field DP. A study of the heterogeneity of plastic deformation in IF steel by EBSD. Materials Science and Engineering: A. 2012;548(30):56-63.

2. Scheriau S, Pippan R. Influence of grain size on orientation changes during plastic deformation. Materials Science and Engineering: A. 2008;493(1-2):48-52.

3. Franciosi P, Berbenni S. Multi-laminate plastic-strain organization for non-uniform TFA modeling of poly-crystal regularized plastic flow. International Journal of Plasticity. 2008;24(9):1549-1580.

4. Kanjarla AK, Van Houtte P, Delannay L. Assessment of plastic heterogeneity in grain interaction models using crystal plasticity finite element method. International Journal of Plasticity. 2010;26(8):1220-1233.

5. Rollett AD, Wagner F, Allain-Bonasso N, Field D, Lebensohn RA. Comparison of Gradients in Orientation and Stress between Experiment and Simulation. Materials Science Forum. 2012;702703:463-468.

6. Soifer YM, Verdyan A, Kazakevich M, Rabkin E. Nanohardness of copper in the vicinity of grain boundaries. Scripta Materialia. 2002;47(12):799-804.

7. Soer WA, Aifantis KE, De Hosson JTM. Incipient plasticity during nanoindentation at grain boundaries in body-centered cubic metals. Acta Materialia. 2005;53(17):4665-4676.

8. Calcagnotto M, Ponge D, Demir E, Raabe D. Orientation gradients and geometrically necessary dislocations in ultrafine grained dual-phase steels studied by 2D and 3D EBSD. Materials Science and Engineering: A. 2010;527(10-11):2738-2746.

9. Takayama Y, Szpunar JA, Kato H. Analysis of intergranular misorientation related to deformation in an Al-Mg-Mn alloy. Materials Science Forum. 2005;495-497:1049-1054.

10. Pouillier E, Gourgues AF, Tanguy D, Busso EP. A study of intergranular fracture in an aluminum alloy due to hydrogen embrittlement. International Journal of Plasticity. 2012;34:139153.

11. Basu I, Ocelík V, De Hosson JTM. Measurement of spatial stress gradients near grain boundaries. Scripta Materialia. 2017;136:11-14.

12. Mishra SK, Pant P, Narasimhan K, Rollett AD, Samajdar I. On the widths of orientation gradient zones adjacent to grain boundaries. Scripta Materialia. 2009;61(3):273-276.

13. Subedi S, Pokharel R, Rollett AD. Orientation gradients in relation to grain boundaries at varying strain level and spatial resolution. Materials Science and Engineering: A. 2015;638:348356.

14. Lee TC, Robertson IM, Birnbaum HK. Prediction of slip transfer mechanisms across grain boundaries. Scripta Metallurgica. 1989;23(5):799-803. 
15. Wo PC, Ngan AHW. Investigation of slip transmission behavior across grain boundaries in polycrystalline Ni3 Al using nanoindentation. Journal of Materials Research. 2004;19(1):189201.

16. Signorelli JW, Roatta A, De Vincentis N, Schwindt C, Avalos M, Bolmaro RE, et al. Electron backscatter diffraction study of orientation gradients at the grain boundaries of a polycrystalline steel sheet deformed along different loading paths. Journal of Applied Crystallography. 2017;50:1179-1191.

17. Aust K, Chen NK. Effect of orientation difference on the plastic deformation of aluminum bicrystals. Acta Metallurgica. 1954;2(4):632-633, 635-638.

18. Sutton AP, Baluffi RW. Interfaces in Crystalline Materials (Oxford Classical Texts in the Physical Sciences). Oxford: Oxford University Press; 2006.

19. Sangid MD, Ezaz T, Sehitoglu H. Energetics of residual dislocations associated with slip-twin and slip-GBs interactions. Materials Science and Engineering: A. 2012;542:21-30.

20. Livingston JD, Chalmers B. Multiple slip in bicrystal deformation. Acta Metallurgica. 1957;5(6):322-327.

21. Shen Z, Wagoner RH, Clark WAT. Dislocation and grain boundary interactions in metals. Acta Metallurgica. 1988;36(12):32313242 .

22. van Beers PRM, McShane GJ, Kouznetsova VG, Geers MGD. Grain boundary interface mechanics in strain gradient crystal plasticity. Journal of the Mechanics and Physics of Solids. 2013;61(12):2659-2679.

23. Abuzaid WZ, Sangid MD, Carroll JD, Sehitoglu H, Lambros J. Slip transfer and plastic strain accumulation across grain boundaries in Hastelloy X. Journal of the Mechanics and Physics of Solids. 2012;60(6):1201-1220.

24. Vachhani SJ, Doherty RD, Kalidindi SR. Studies of grain boundary regions in deformed polycrystalline aluminum using spherical nanoindentation. International Journal of Plasticity. 2016;81:87101.

25. Clark WAT, Wagoner RH, Shen ZY, Lee TC, Robertson IM, Birnbaum HK. On the criteria for slip transmission across interfaces in polycrystals. Scripta Metallurgica et Materialia. 1992;26(2):203206.

26. Luster J, Morris MA. Compatibility of deformation in two-phase Ti-Al alloys: Dependence on microstructure and orientation relationships. Metallurgical and Materials Transactions A. 1995;26(7):1745-1756.

27. Bieler TR, Eisenlohr P, Zhang C, Phukan HJ, Crimp MA. Grain boundaries and interfaces in slip transfer. Current Opinion in Solid State and Materials Science. 2014;18(4):212-226.
28. Konijnenberg PJ,Zaefferer A, Raabe D. Assessment of geometrically necessary dislocation levels derived by 3D EBSD. Acta Materialia. 2015;99:402-414.

29. Mingard KP, Roebuck B, Jones HG, Stewart M, Cox D, Gee MG. Visualisation and measurement of hardmetal microstructures in 3D. International Journal of Refractory Metals and Hard Materials. 2018;71:285-291.

30. Kamaya M. Assessment of local deformation using EBSD: Quantification of local damage at grain boundaries. Materials Characterization. 2012;66:56-67.

31. Wagner F, Ouarem A, Gu CF, Allain-Bonasso N, Toth LS. A new method to determine plastic deformation at the grain scale. Materials Characterization. 2014;92:106-117.

32. Hachim A, El Ghorba M, Hariri S, Akef A. Numerical evaluation of stress triaxiality at the top of notch for a specimen steel notched bi-S355. International Journal of Engineering and Science. 2012;1(1):58-64.

33. Lindner D, Mathieu F, Hild F, Allix O, Minh CH, Paulien-Camy O. On the evaluation of stress triaxiality fields in a notched titanium alloy sample via integrated DIC. Journal of Applied Mechanics. 2015;82(7):4030457.

34. Kamaya M. Assessment of local deformation using EBSD: Quantification of accuracy of measurement and definition of local gradient. Ultramicroscopy. 2011;111(8)1189-1199.

\section{Supplementary material}

The following online material is available for this article: Table S1: shows information regarding the initial conditions of the grains and GBs analyzed in the paper.

Figure S1: Misorientation profiles obtained from GBs $\mathrm{AE}-\mathrm{BE}$ and BE - DE.

Figure S2: Permeability factors corresponding to GBs $\mathrm{AE}-\mathrm{BE}$ and BE - DE.

Figure S3: IPF maps obtained from the closest zone to EZ inside the notch (nEZ).

Figure S4: Misorientation profiles obtained in selected GBs in nEZ.

Figure S5: Permeability factors obtained for GBs in $\mathrm{nEZ}$. 